\title{
The technology of the superficial chemical and thermal hardening of metal products
}

\section{Mini review}

The wear of conjugation of machines and mechanisms causes a huge damage in all industries. Laser and plasma technologies, ionic processing of metals is applied as measures of fight against it. However, they have not found broad application because of the high cost of the equipment, toxic bursts, need of high qualification of serving personnel in case of slight increase of wear resistance, hardly covering the expenses on their application.

The analysis of structures and expenses of laser processing of metals shows the reasons limiting its application. The structural transformations in metal have no time to be completed because of high speeds of heating and cooling. As a result in structure not in time to be dissolved initial components of ferrite, a perlite, cementitis, residual austenite, a marten site of transformation and all its versions are remained.

The created highly hard conglomerate with high in homogeneity of distribution of carbon does not allow realizing the wear resistance reserves which are available in metal. Structures with low carbon content are a source of the incipient state of surface degradation turning further into avalanche process. Wear resistance after laser processing increases by 1,8-2 times.

The electromechanical processing (EMP) is the other way to increase the wear resistance. The contact durability of the normalized steel after EMP increases by 10-12 times, hardness - by 3,6 times. The same parameters of hardened steel after EMP are increased by $12 \%$ and by 1,66 times respectively. ${ }^{1}$

The amount of the fragmented residual austenite increases in the process of working. The wear resistance significantly increases with increase in content of carbon. The direct dependence between the hardness and wear resistance is not established. At electromechanical processing of samples hardness increases by 3 times, wear resistanceby 9 times. Impact on the wear resistance in these cases is exerted by structure, dispersion, its composition, a ratio of components, direct and the inverse transformation. The provided data once again confirm the existence in metal of big unrealized reserves.

The thermo mechanical influence, the high-speed heating, the cooling, the deformation promote the further crushing of grain of austenite, slow down its growth and constrain its transformation. The hardening is caused by strengthening of all communications between atoms. At the same time there is a change of atomic radiuses, interplane distances, the periods of a crystal lattice that causes strong changes. The microhardness increases to 9000-13000 MPas. ${ }^{1}$

In the technology offered by authors at parameters of thermal influence, commensurable with laser, it was succeeded to slow down process of crystallization of the melted-off zone, to provide completeness of transformation of initial structures and to keep high percent of austenite, having slowed down its transformation.

As a result of high-energy local influence from specially created heating source with a high density of power and high capacities of
Volume 2 Issue 4 - 2017

\author{
Zagorsky Ya V, Zagorsky VK, Kusova I \\ Department of Petroleum engineering, Ufa State Oil Technical \\ University, Russia
}

Correspondence: Zagorsky VK, Department of Petroleum engineering, Ufa State Oil Technical University, Russia, Email kalkutta@mail.ru

Received: February 14, 2017| Published: May 18, 2017

electric and concentration fields (at the expense of the alloying components) the narrow area of the modes of chemical and thermal influence providing homogeneous high-disperse structures is found. All carbon is transferred to a solid solution, promotes stabilization of austenite which content on the separate modes reaches $70-80 \%$ with a micro hardness of 9000-13000 MPas. At a paradoxical combination of a high level of durability, plasticity, viscosity and hardness is simultaneous, with dispersion of structure in the nanodimensional range. Carbides in structure are absent.

The absence of the carbides in the strengthened layer having high abrasive ability, which are wearing out both details of interface by wear products, provided substantial increase of wear resistance of both elements of contact couple.

The electronic microscopy of the strengthened zone at increases in X4000 showed a significant amount of disperse allocations of a germinal phase of the globulyarny graphite playing a role of molecular lubricant at wear.

The electronic microscopy of the strengthened zone at increases in X4000 showed a significant amount of disperse apportionments of a germinal phase of the globulyarny graphite playing a role of molecular lubricant at wear. At the same time it should be noted that highly dispersive austenite in a frictional units plays a role of dry lubricant. The laboratory tests of the strengthened surface in the conditions of a dry friction of sliding according to the scheme a diskpalets showed that the relative wear resistance after hardening increases on the average by two orders in comparison with a reference state. The maximal firmness in the specified conditions is observed on examples with the maximal hardness at the moderate content of residual austenite (to $30 \%$ ). Measurements of temperature in a zone of contact showed its decrease on the strengthened surface by $4-5$ times and a friction coefficient - by 1,5-2 times.

Bench tests of exemplars in the conditions of rigid abrasive, hydro abrasive, cavitational, frictional destruction showed increase in wear resistance up to 10 times.

The electronic translucent microscopy by method of foil at increases x 100000-200000 has shown high dispersion of thin structure of austenite within 5-10 Nanometers and more and the existence of light spots in graphite inclusions. In works ${ }^{2,3}$ it is suggested that light spots 
have the fullerene nature. This fact has formed the basis for carrying out spectral researches regarding existence in the strengthened layer of fullerenes.

As a result of spectral analysis with IK-Fourier spectrometer Avatar E. S. P. of the hardened sample by a special technique the characteristic peaks of $\mathrm{C} 60$ its derivatives and isomers were found. After processing the hardened samples by a hydrofluoric acid were compared the dried-up suspension with a spectrum of fullerenes of high purity of $99,7 \%$ that allowed to detect the formation the or derivative of C60 fullerenes. After the treatment with tetrachloride carbon the peaks in the field of frequencies of chlorderivative fullerenes were found.

The carried out researches allow to explain the high wear resistance of the hardened layers, the reasons of which are: the high percentage content of austenite, the transformation, the absence of carbides, the presence of inclusions of high-dispersed graphite and very hard spheroidal fullerenes C60 and their modifications at the nanoscale the structure of the thin structure.

The combination of the mechanisms of nanostructural structure and characteristics of processes occurring during the wear in the thin surface layers associated with the transformation of high carbon austenite to martensite, contributed to a significant increase of resistance to the destructive action of the environment and reduction of wear to the level, after which begins a catastrophic failure of the surface due to fatigue spall.

The industrial tests of a wide range of parts operating under conditions of extreme wear - the fingers of the dredgers, bandages of diesel locomotives, electric locomotives, trams, and other details, confirmed the situation and the results presented in this article.

\section{Acknowledgements}

None.

\section{Conflict of interest}

The author declares no conflict of interest.

\section{References}

1. Askinazi BM. Hardening and recovery of parts of electromechanical processing. Mashinostroyeniye. Moscow, 1989.

2. Zhukov AA. The forms of existence of carbon in cast iron. 1992.

3. Lubchenco AP. The buckyballs. Steady germs of spherical grains of graphite. 1992 